\title{
Is the Correct Anatomical Placement of the Electrocardiogram (ECG) Electrodes Essential to Diagnosis in the Clinical Setting A Systematic Review
}

Alexandros Stavros Hadjiantoni ( $\nabla$ ahadjiantoni@nhs.net )

The University of the West of England (UWE)

\section{Research article}

Keywords: Electrocardiogram, Electrode, Cardiology, Lead, Anatomy, Systematic Review

Posted Date: September 22nd, 2020

DOI: https://doi.org/10.21203/rs.3.rs-74147/v1

License: (1) This work is licensed under a Creative Commons Attribution 4.0 International License. Read Full License

Version of Record: A version of this preprint was published at Cardiology and Cardiovascular Medicine on January 1st, 2021. See the published version at https://doi.org/10.26502/fccm.92920135. 


\section{Abstract}

Background: Anatomical misplacement of the Electrocardiogram (ECG) electrode(s) is common, with significant impact on clinical diagnosis. Reasons are multi-faceted, with this review examining the consequential effects of misplacement to ECG morphology, diagnosis, prognosis, patient outcomes, and potential impact to patient care pathway.

Objectives: This review examined the significance of misplacement, its' commonality and ensuing effect on patient safety, accurate ECG acquisition and diagnosis, with evaluation of reasons for such misplacement.

Methodology: Review of available literature was conducted using electronic databases. In-line with the Preferred Reporting Items for Systematic reviews and Meta - analysis protocols (PRISMA) 2015 checklist, this review was conducted with search criteria, search terms, eligibility for inclusion/ exclusion criteria, extraction and data analysis predetermined by the authors. Keywords were arranged according to grouping of terms surrounding ECG, anatomical placement, and diagnosis. The search strategy was conducted during September/October 2019. Scoping searches were conducted alongside reference lists of included studies hand searched (Snowballing) for further relevant studies. The Critical Appraisal Skills Programme (CASP) was used to methodically appraise papers (CASP, 2019). Screening of titles and abstracts of identified citations was performed by a single reviewer. Eligible articles then full text screened independently by two reviewers. Disagreements were discussed and resolved by a third reviewer. In instances of unclear reporting, authors were contacted to provide further information and clarity. Assessment of relevant literature and critical appraisal of primary research, pertaining to the clinical diagnosis and effects of anatomical misplacement of ECG electrodes, formulate the thematic discussion drawn by this review.

Results: This review identified a plethora of causes, ranging from: operator error; lack of anatomical awareness; inaccurate assessment of anatomical landmarks; obesity; differences in anatomy/gender; levels of undress and lack of appreciation of consequences of misplacement, both modifiable and nonmodifiable attributable to electrode misplacement. Clinical diagnosis can be altered owing to erroneous placement of electrodes. ECG morphology is altered due to incorrect anatomical misplacement, culminating substandard practice, a missed diagnosis or misdiagnosis and potential harm.

Conclusion: Correct anatomical placement of ECG electrodes is essential to diagnosis in the clinical setting. Peer-led educational intervention with mandatory training is essential to improve practice.

\section{PROSPERO Registration Number: CRD42019152461}

\section{Background}

Prevalence of Coronary Artery Disease: Coronary artery disease (CAD) resides as the leading cause of death in most countries worldwide. ${ }^{[1]}$ CAD and coronary heart disease (CHD) are synonymous, and terms which are used interchangeably. A heart attack is often the result of a build-up of plaque in the heart's arteries, or coronary arteries. The coronary arteries supply blood to the heart and if this flow of blood becomes limited due to a build-up of plaque, ischemia ensues. This means that the heart's cells and muscle are starved of oxygenated, nutrient rich blood. If undiagnosed and left untreated, chronic ischemia and progressive narrowing of the coronaries lead to injury and infarct, or death of the cells and muscle of the heart. CAD has life threatening characteristics, with warning signs such as chest pain, shortness of breath (SOB) and palpitations. Therefore, rapid decision-making and diagnosis is essential.

The current recognised gold standard for assessment and identification of CAD is Angiography. However, there are associated risks and possible complications with this invasive modality. There is evidence that CAD and plaques are formed in childhood, yet CAD disease is preventable. ${ }^{[2]}$ Typical risk factors include smoking, diabetes, high blood pressure (hypertension), family history, obesity and poor life style choices.

The standard 12 lead electrocardiogram (ECG) provides for a non-invasive, cost effective assessment that is convenient to patients. ${ }^{[3]}$ The ECG, being the assessment of the electrophysiological activity of the heart over time, is to date the most frequently requested and conducted diagnostic/prognostic tool within the clinical setting. ${ }^{[4][5][6]}$ Its' fundamental morphological features provide useful information in the diagnosis/prognosis of arrhythmia and cardiac disease. ${ }^{[7][8]}$ Accurate electrode placement and acquisition of ECG is an essential skill required of nursing staff and health professionals alike and duly noted by The Nursing and Midwifery Council (NMC) (2010) standards for preregistration nurses. ${ }^{[9]}$

Principally, acquisition of ECG traces should be reproducible and correctly identify various medical conditions such as cardiac arrhythmia, particularly acute coronary syndromes (ACS), and which aid in risk stratification, detection and management of cardiac disease, notably ST elevation myocardial infarction. ${ }^{[10][6]}$

This graphical representation of the signal potential difference between two points provides for universal standard parameters of the ECG waveform. ${ }^{\text {[11] }}$ Typically depicted as the P-wave (Atrial depolarization, with subsequent contraction), the QRS complex (Ventricular depolarization, with subsequent contraction), and the T-wave and U-wave which constitute the repolarisation phase of the cardiac cycle. ${ }^{[12]}$ Any deviation from the perceived normal ECG baseline requires accurate and reliable detection owing to time/frequency varying morphology.

It follows that, acquisition, recognition, analysis, diagnosis and subsequent treatment of patients is, in part, reliant on this non-invasive electro-cardiology procedure. ${ }^{[13][14]}$ Regarded as the 'gold standard,' a non-invasive, inexpensive diagnostic tool, integral in medical investigations, the electrocardiogram remains the principal diagnostic method in the recognition of acute myocardial infarction (MI), British Cardiovascular Society (BSC) (2015) and National Institute for Health and Care Excellence (NICE) (2016). ${ }^{[15][16]}$

Historically, substandard levels have prevailed in the acquisition of ECG, with $50 \%$ of nurses and $<20 \%$ of cardiologists correctly placing precordial electrodes V1 and V2. ${ }^{[17]}$ This erroneous pervasiveness continues in Today's practice. These findings were supported by Medani et al, (2018), ${ }^{[14]}$ which revealed nurses 
misplaced precordial electrodes in $64 \%$ of cases. Misplacement constitutes for erroneous acquisition, diagnosis, missed diagnosis, interpretation, and treatment/mistreatment of patients. ${ }^{[18]}$ Furthermore, delayed triage to early invasive strategy in the patient care pathway has resulted from erroneous placement, with consequent increased mortality and morbidity. ${ }^{[18]}$ The leading cause of mortality and serious illness to date remains cardiovascular disease. ${ }^{[6]}$

The prevalence of electrode misplacement is a common finding in both acute and clinical settings. ${ }^{[13][14]}$ Despite the commonality of misplacement, such erroneous practice remains under reported. Of paramount importance in any attempt to acquire an ECG trace that is accurate and of good reproducible quality, is the onus placed on correct anatomical placement of the silver-silver chloride electrodes and their relative leads. The respected 'gold standard' should therefore consider the recommendations and guidelines of the Society for Cardiological Science and Technology (SCST) adherence to which remains widely neglected in clinical practice. ${ }^{[19]}$

The electrocardiogram has changed little since its inception, with the overarching prerequisite for any accurate detection of signal remaining the accurate

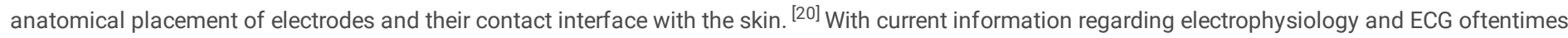
disjointed, contradictory and frequently misunderstood, particularly in an era of multi-authored texts, the need for a single authoritative voice is essential. ${ }^{[6]}$

The SCST recommend standardization of the ECG procedure, with training and subsequent accreditation, ensuring operator skill, competency, confidence, and a gold standard of practice and promotion of excellence. ${ }^{[19]}$

\section{Objectives:}

This systematic review investigated the significant importance of correct anatomical placement of the electrocardiogram (ECG) electrodes and their relative leads. Objectives were made to establish the commonality of electrode misplacement, its' subsequent potential effect on patient safety and a review of reasons for such misplacement.

\section{Design And Methodology}

Initially, the review sought to highlight the advancements in technology, which provide for signal processing of the electrocardiogram, notably, differences in Fourier Transform (FT), and Continuous Wavelet Transform (CWT). However, the caveat adopted by this review acknowledges the absence in the literature of any direct comparison between the modalities and subsequent paucity of literature to warrant expansion.

Consideration was therefore given to the common denominator governing both signal processing modalities. In short, both are reliant upon the interface between the patients' skin and the silver-silver chloride electrodes with their respective leads, for the attainment of the surface 12 - Lead ECG.

\section{Justification:}

A risk of bias assessment was conducted for each of the included studies using the Critical Appraisal Skills Programme (CASP) (2018). ${ }^{[21]}$ The population, intervention, comparison and outcome (PICO) framework (NICE, 2012) for eligibility criteria are described as follows:

\section{Population}

Male or female adults of $\geq 18$ years, regardless of any previous cardiac history were considered.

\section{Intervention}

Electrode placement with the sole intention to acquire a 12 - Lead electrocardiogram trace for clinical diagnosis of arrhythmia.

\section{Comparison}

Accurate anatomical placement of ECG electrodes in-line with

guidelines and recommendations of the (SCST, 2017) compared with those misplaced.

\section{Outcome}

The potential effect(s) on patients' clinical diagnosis, owing to inaccurate ECG electrode placement.

In accordance with the Nursing and Midwifery Code of Conduct (NMC, 2015), ${ }^{[22]}$ and guidelines/recommendations of the SCST ${ }^{[19]}$ justification for this work is demonstration of the ongoing prevalence and relevance of ECG electrode misplacement in the clinical setting. Furthermore, the subsequent ramifications imposed on patient diagnosis and implications of erroneous ECG acquisition.

\section{Search Strategy:}


A systematic review was conducted with search criteria, search terms, eligibility for inclusion/exclusion criteria, extraction and analysis of data predetermined by the authors, in-line with the Preferred Reporting Items for Systematic reviews and Meta - analysis protocols (PRISMA) 2015 checklist. ${ }^{[23]}$ Keywords and subject headings were combined according for terms surrounding, ECG, anatomical placement, and diagnosis. Table 1 highlights the systematic review synthesis and data extraction. No time/date restrictions of the literature were implemented. The inclusion criteria for this review included English language articles, international papers, and unpublished works. 
Table 1

Systematic Review Synthesis and Data Extraction

\begin{tabular}{|c|c|c|c|c|c|c|c|}
\hline Author & Design & Method & Reason & $\begin{array}{l}\text { Misplaced } \\
\text { Electrode } \\
\text { (Most } \\
\text { Common } \\
\text { listed first) }\end{array}$ & $\begin{array}{l}\text { Clinical Significance } \\
\text { of Misplacement }\end{array}$ & Result & Recommenc \\
\hline $\begin{array}{l}\text { Rajaganeshan } \\
\text { et al, } 2007\end{array}$ & $\begin{array}{l}\text { Multi-center } \\
\text { Prospective } \\
\text { cohort study, } \\
\text { UK }\end{array}$ & $\begin{array}{l}119 \text { medical } \\
\text { personnel } \\
\text { acquiring } 12 \\
\text { lead ECG, } \\
\text { Doctors, } \\
\text { Nurses, } \\
\text { Technicians, } \\
\text { Physicians, } \\
\text { cardiologists }\end{array}$ & $\begin{array}{l}\text { Operator Error, } \\
\text { anatomical differences, } \\
\text { obesity }\end{array}$ & $\begin{array}{l}\text { V1; V2; V4; } \\
\text { V5; V6 }\end{array}$ & $\begin{array}{l}\text { Potentially harmful } \\
\text { treatment/therapeutic } \\
\text { procedures. } \\
\text { Misclassifying } \\
\text { ischemic changes }\end{array}$ & $\begin{array}{l}\text { Wide inter- } \\
\text { individual variation } \\
\text { in placement }\end{array}$ & $\begin{array}{l}\text { Training/edı } \\
\text { regarding co } \\
\text { identificatior } \\
\text { anatomical } \\
\text { landmarks, } \\
\text { positioning c } \\
\text { electrodes a } \\
\text { clinical } \\
\text { implications }\end{array}$ \\
\hline $\begin{array}{l}\text { Wallen et al, } \\
2013\end{array}$ & $\begin{array}{l}\text { Paramedic } \\
\text { ECG } \\
\text { acquisition, } \\
\text { New Zealand }\end{array}$ & $\begin{array}{l}50 \text { Female } \\
\text { participants } \\
\text { (Multi } \\
\text { ethnicity) on } \\
\text { and under the } \\
\text { breast }\end{array}$ & $\begin{array}{l}\text { Anatomical } \\
\text { differences, Breast } \\
\text { tissue }\end{array}$ & $\begin{array}{l}\text { Modified } \\
\text { placement } \\
\text { owing to } \\
\text { gender. } \\
\text { Paramedics } \\
\text { attitude to } \\
\text { ECG } \\
\text { acquisition } \\
\text { with } \\
\text { hesitance } \\
\text { to expose } \\
\text { female } \\
\text { chest }\end{array}$ & $\begin{array}{l}\text { Women with ACS } \\
\text { potentially receive } \\
\text { fewer cardiac } \\
\text { investigations and } \\
\text { less treatment than } \\
\text { men }\end{array}$ & $\begin{array}{l}26 \text { women }(52 \%) \\
\text { preferred } \\
\text { electrodes placed } \\
\text { on breast, } 19(38 \%) \\
\text { were indifferent } \\
\text { and } 5(10 \%) \\
\text { preferred under } \\
\text { breast }\end{array}$ & $\begin{array}{l}\text { Further rese: } \\
\text { into views ol } \\
\text { women with } \\
\text { cultural diff } \\
\text { perspectives } \\
\text { regarding el } \\
\text { placement o } \\
\text { breast tissue }\end{array}$ \\
\hline $\begin{array}{l}\text { Davis et al, } \\
2018\end{array}$ & $\begin{array}{l}\text { PULSE, Multi- } \\
\text { center } \\
\text { Randomised } \\
\text { clinical trial, } \\
\text { USA, Canada } \\
\text { and China }\end{array}$ & $\begin{array}{l}2,956 \text { Patients } \\
\text { (42\% Female) } \\
\text { from cardiac } \\
\text { units in the } \\
\text { USA, Canada } \\
\text { and China. } \\
\text { ECG } \\
\text { monitoring } \\
\text { education } \\
\text { programme } \\
\text { implementing } \\
\text { AHA practice } \\
\text { standards }\end{array}$ & $\begin{array}{l}\text { Anatomical } \\
\text { differences, gender, } \\
\text { accuracy of precordial } \\
\text { electrode placement }\end{array}$ & $\begin{array}{l}\text { Precordial } \\
\text { electrodes } \\
\text { V1-V6 }\end{array}$ & $\begin{array}{l}\text { Alteration of } \\
\text { waveform } \\
\text { morphology, leading } \\
\text { to misdiagnosis of } \\
\text { arrhythmias and } \\
\text { ischemia }\end{array}$ & $\begin{array}{l}\text { Precordial (V-lead) } \\
\text { placement did not } \\
\text { differ by gender, } \\
\text { however, <50\% of } \\
\text { men and women } \\
\text { assessed had } \\
\text { accurate electrode } \\
\text { placement }\end{array}$ & $\begin{array}{l}\text { Online educ: } \\
\text { and strategii } \\
\text { change prac } \\
\text { improve nur: } \\
\text { knowledge a } \\
\text { QoC regardir } \\
\text { ECG. Signific } \\
\text { improvemen } \\
\text { concluded w } \\
\text { implementa } \\
\text { AHA practice } \\
\text { standards }\end{array}$ \\
\hline $\begin{array}{l}\text { Day et al, } \\
2015\end{array}$ & $\begin{array}{l}\text { Single Centre } \\
\text { retrospective } \\
\text { study, USA }\end{array}$ & $\begin{array}{l}55 \text { Patients } 32 \\
(58 \%) \text { Male, } 23 \\
(42 \%) \text { Female, } \\
\text { each received } \\
\text { chest } \\
\text { computer } \\
\text { tomography } \\
\text { (CT) } \\
\text { examination, } \\
\text { reviewed for } \\
\text { measurements } \\
\text { to correctly } \\
\text { identify } 4^{\text {th }} \\
\text { intercostal } \\
\text { space for } \\
\text { accurate } \\
\text { electrode } \\
\text { placement }\end{array}$ & $\begin{array}{l}\text { Obese patients, } \\
\text { inaccurate } \\
\text { palpation/identification } \\
\text { of intercostal spaces }\end{array}$ & $\begin{array}{l}\text { Sternal } \\
\text { notch; } \\
\text { Xiphoid } \\
\text { process; } 4^{\text {th }} \\
\text { Intercostal } \\
\text { space; V1 }\end{array}$ & $\begin{array}{l}\text { Misinterpretation/ } \\
\text { incorrect diagnosis of } \\
\text { anterior infarction, } \\
\text { anteroseptal } \\
\text { infarction, ventricular } \\
\text { hypertrophy, } \\
\text { ischemia, Brugada } \\
\text { syndrome }\end{array}$ & $\begin{array}{l}\text { The measurement } \\
\text { identified by the } \\
\text { study may be } \\
\text { utilized to identify } \\
\text { the } 4^{\text {th }} \text { intercostal } \\
\text { space for accurate } \\
\text { placement of } \\
\text { precordial } \\
\text { electrodes }\end{array}$ & $\begin{array}{l}\text { Use of the st } \\
\text { notch and X } \\
\text { process, eve } \\
\text { obese peopl } \\
\text { reference po } \\
\text { accurately ic } \\
\text { intercostal s } \\
\text { essential as } \\
\text { remaining } \\
\text { precordial } \\
\text { electrodes a } \\
\text { placed base } \\
\text { this initial } \\
\text { electrode } \\
\text { placement }\end{array}$ \\
\hline $\begin{array}{l}\text { Medani et al, } \\
2018\end{array}$ & $\begin{array}{l}\text { Prospective } \\
\text { pre- and post- } \\
\text { intervention } \\
\text { performance } \\
\text { analysis } \\
\text { study, Ireland }\end{array}$ & $\begin{array}{l}100 \text { medical } \\
\text { personnel, } \\
\text { Doctors, } \\
\text { Nurses, } \\
\text { Technicians, } \\
\text { randomly } \\
\text { selected to } \\
\text { place sticker } \\
\text { dots on } \\
\text { mannequin, } \\
\text { recorded on } \\
\text { radar plot and } \\
\text { compared to } \\
\text { correct } \\
\text { precordial } \\
\text { positions }\end{array}$ & $\begin{array}{l}\text { To asses competence } \\
\text { of placement, and } \\
\text { improve performance } \\
\text { through a peer-led } \\
\text { educational } \\
\text { intervention }\end{array}$ & $\begin{array}{l}\text { Placing V1 } \\
\text { and V2 too } \\
\text { superiorly } \\
\text { and V5 and } \\
\text { V6 too } \\
\text { medially }\end{array}$ & $\begin{array}{l}\text { Erroneous diagnosis, } \\
\text { poor R wave } \\
\text { progression, poor } \\
\text { reproducibility of ECG } \\
\text { amplitude } \\
\text { measurement, } \\
\text { anteroseptal } \\
\text { infarction and } \\
\text { ventricular } \\
\text { hypertrophy, } \\
\text { alterations to QRS } \\
\text { complex and T wave } \\
\text { prominence and } \\
\text { morphology. False } \\
\text { positive and false } \\
\text { negative ischemic } \\
\text { changes on ECG }\end{array}$ & $\begin{array}{l}\text { Placing V1 and V2 } \\
\text { too superiorly and } \\
\text { V5 and V6 too } \\
\text { medially. } \\
\text { Significant } \\
\text { increase in } \\
\text { accuracy post } \\
\text { intervention with } \\
80-85 \% \\
\text { homogeneity } \\
\text { achieved compared } \\
\text { to } 34 \% \text { on initial } \\
\text { assessment }\end{array}$ & $\begin{array}{l}\text { Educational } \\
\text { intervention, } \\
\text { periodic retr: } \\
\text { peer-led trair } \\
\text { either six-me } \\
\text { or on an ann } \\
\text { basis }\end{array}$ \\
\hline Walsh, 2018 & $\begin{array}{l}5 \times \text { Case } \\
\text { Presentations }\end{array}$ & $\begin{array}{l}\text { Case 1: } \\
\text { Patient } \\
\text { presented to } \\
\text { ED }\end{array}$ & $\begin{array}{l}\text { Multi-factorial: V1 and } \\
\text { V2 misplaced } \\
\text { superiorly }\end{array}$ & $\begin{array}{l}\text { V1 and V2 } \\
\text { misplaced } \\
\text { superiorly }\end{array}$ & $\begin{array}{l}\text { Misplacement of V1 } \\
\text { and V2 can produce } \\
\text { false poor } r \text { wave } \\
\text { progression, false } \\
\text { Incomplete Right } \\
\text { Bundle Branch Block }\end{array}$ & $\begin{array}{l}\text { Cases identify the } \\
\text { potential clinical } \\
\text { ramifications } \\
\text { associated with } \\
\text { misplacement of } \\
\text { precordial }\end{array}$ & $\begin{array}{l}\text { Awareness c } \\
\text { misplaceme }\end{array}$ \\
\hline
\end{tabular}

Page 5/13 


\begin{tabular}{|c|c|c|c|c|c|c|c|}
\hline & & $\begin{array}{l}\text { Case 2: } \\
\text { Patient } \\
\text { presented to } \\
\text { primary care } \\
\text { physician } \\
\text { Case 3: } \\
\text { Patient } \\
\text { presented for } \\
\text { routine } \\
\text { medical } \\
\text { evaluation } \\
\text { Case 4: } \\
\text { Patient } \\
\text { presented to } \\
\text { primary care } \\
\text { physician } \\
\text { Case 5: } \\
\text { Patient } \\
\text { presented to } \\
\text { ED }\end{array}$ & $\begin{array}{l}\text { Obesity, clothing not } \\
\text { removed, lead } \\
\text { switching }\end{array}$ & & $\begin{array}{l}\text { (IRBBB), can suggest } \\
\text { pulmonary embolism, } \\
\text { Type } 2 \text { Brugada, } \\
\text { anterior ST segment } \\
\text { elevation, anterior T } \\
\text { wave inversion }\end{array}$ & $\begin{array}{l}\text { electrodes and } \\
\text { leads }\end{array}$ & \\
\hline $\begin{array}{l}\text { McCann et al, } \\
2007\end{array}$ & $\begin{array}{l}\text { Prospective } \\
\text { observational } \\
\text { study }\end{array}$ & $\begin{array}{l}\text { Assessment of } \\
\text { ECG electrode } \\
\text { placement in } \\
77 \text { patients as } \\
\text { part of routine } \\
\text { ED care }\end{array}$ & Operator error, & $\begin{array}{l}\text { Wide } \\
\text { spread } \\
\text { inter- } \\
\text { operator } \\
\text { electrode } \\
\text { placement } \\
\text { variation, } \\
\text { particularly } \\
\text { lateral } \\
\text { leads }\end{array}$ & $\begin{array}{l}\text { Greater lateral chest } \\
\text { electrode discordance } \\
\text { noted for both male } \\
\text { and female patients, } \\
\text { than those of central } \\
\text { chest electrodes. } \\
\text { Electrode } \\
\text { misplacement of } \\
20 \text { mm or more is } \\
\text { associated with } \\
\text { significant QRS } \\
\text { morphology that may } \\
\text { affect clinical } \\
\text { interpretation }\end{array}$ & $\begin{array}{l}\text { Inter-operator } \\
\text { variability. } \\
\text { Physical } \\
\text { identification of } \\
\text { defined anatomical } \\
\text { landmarks is an } \\
\text { inexact science. } \\
\text { Patient factors } \\
\text { may further impede } \\
\text { accurate location, } \\
\text { particularly in older } \\
\text { larger women. } \\
\text { Even expert } \\
\text { assessment of } \\
\text { correct ECG } \\
\text { electrode } \\
\text { location/placement } \\
\text { is not a reliable } \\
\text { reference standard }\end{array}$ & $\begin{array}{l}\text { Leaving che } \\
\text { electrodes in } \\
\text { throughout t } \\
\text { patient's hos } \\
\text { stay may he } \\
\text { minimize } \\
\text { variability, } \\
\text { however, this } \\
\text { be uncomfo } \\
\text { impracticabl }\end{array}$ \\
\hline
\end{tabular}

The study is reported and conducted according to the Preferred Reporting Items for Systematic reviews and Meta-Analyses (PRISMA) guidelines. The systematic review protocol was prospectively registered on the PROSPERO database of systematic reviews, Registration Number: CRD42019152461, and inline with the Preferred Reporting Items for Systematic reviews and Meta-Analyses Protocols (PRISMA-P), (2015) checklist. Critical Appraisal Skills Programme (CASP) was used to methodically appraise papers (CASP, 2019). ${ }^{\text {[21] }}$

The search strategy was developed and conducted during September and October 2019. A total of three (3) scoping searches were conducted along with the reference lists of included studies hand searched (Snowballing) for further relevant studies. A copy of the Healthcare Databases Advanced Search (HDAS) NICE ${ }^{[24]}$ search strategy is included in Table 2. 
Healthcare Databases Advanced Search (HDAS) NICE search strategy (Electronic Databases)

\begin{tabular}{|c|c|c|c|}
\hline \# & Database & Search term & Results \\
\hline 1 & Medline & (ecg).ti,ab & 60247 \\
\hline 2 & Medline & (electrocardiogram).ti,ab & 36766 \\
\hline 3 & Medline & ELECTROCARDIOGRAPHY/ & 186536 \\
\hline 22 & Medline & (electrocardiograph*).ti,ab & 44904 \\
\hline 23 & Medline & (1 OR 2 OR 3 OR 22) & 235131 \\
\hline 5 & Medline & $\left(\right.$ electrode $\left.{ }^{\star}\right) . t i, a b$ & 147008 \\
\hline 6 & Medline & ELECTRODES/ & 45184 \\
\hline 7 & Medline & (5 OR 6) & 163150 \\
\hline 8 & Medline & (placement).ti,ab & 121217 \\
\hline 9 & Medline & $($ position*).ti,ab & 567725 \\
\hline 10 & Medline & (anatomical).ti,ab & 139469 \\
\hline 12 & Medline & (misplacement).ti,ab & 1725 \\
\hline 24 & Medline & (Angle of louis).ti,ab & 41 \\
\hline 26 & Medline & (malposition).ti,ab & 4122 \\
\hline 29 & Medline & $($ misposition*).ti,ab & 281 \\
\hline 30 & Medline & (mis-position*).ti,ab & 30 \\
\hline 31 & Medline & (misplaced OR mis-placed).ti,ab & 2405 \\
\hline 32 & Medline & $($ error*).ti,ab & 285306 \\
\hline 33 & Medline & "MEDICAL ERRORS"/ OR exp "DIAGNOSTIC ERRORS"/ & 129236 \\
\hline 34 & Medline & (misdiagnos* OR mis-diagnos*).ti,ab & 31019 \\
\hline 35 & Medline & (8 OR 9 OR 10 OR 12 OR 24 OR 26 OR 29 OR 30 OR 31 OR 32 OR 33 OR 34) & 1202184 \\
\hline 36 & Medline & (23 AND 7 AND 35) & 1375 \\
\hline 37 & EMBASE & (ecg).ti,ab & 103111 \\
\hline 38 & EMBASE & (electrocardiogram).ti,ab & 47724 \\
\hline 39 & EMBASE & ELECTROCARDIOGRAM/ & 114739 \\
\hline 40 & EMBASE & (electrocardiograph*).ti,ab & 51551 \\
\hline 41 & EMBASE & (37 OR 38 OR 39 OR 40) & 216323 \\
\hline 42 & EMBASE & $\left(\right.$ electrode $\left.^{\star}\right) . t i, a b$ & 165348 \\
\hline 43 & EMBASE & ELECTRODE/ & 101835 \\
\hline 44 & EMBASE & (42 OR 43) & 185992 \\
\hline 45 & EMBASE & (placement).ti,ab & 173569 \\
\hline 46 & EMBASE & (position*).ti,ab & 673332 \\
\hline 47 & EMBASE & (anatomical).ti,ab & 188133 \\
\hline 48 & EMBASE & (misplacement).ti,ab & 2399 \\
\hline 49 & EMBASE & (Angle of louis).ti,ab & 31 \\
\hline 50 & EMBASE & (malposition).ti,ab & 5477 \\
\hline 51 & EMBASE & $($ misposition*).ti,ab & 381 \\
\hline 52 & EMBASE & (mis-position*).ti,ab & 55 \\
\hline 53 & EMBASE & (misplaced OR mis-placed).ti,ab & 3209 \\
\hline 54 & EMBASE & $($ error*).ti,ab & 357058 \\
\hline 55 & EMBASE & "MEDICAL ERROR"/ OR exp "DIAGNOSTIC ERROR"/ & 110041 \\
\hline 56 & EMBASE & (misdiagnos* OR mis-diagnos*).ti,ab & 45090 \\
\hline
\end{tabular}




\begin{tabular}{|c|c|c|c|}
\hline 57 & EMBASE & (45 OR 46 OR 47 OR 48 OR 49 OR 50 OR 51 OR 52 OR 53 OR 54 OR 55 OR 56) & 1443769 \\
\hline 58 & EMBASE & (41 AND 44 AND 57) & 1416 \\
\hline 59 & CINAHL & (ecg).ti,ab & 12052 \\
\hline 60 & CINAHL & (electrocardiogram).ti,ab & 8212 \\
\hline 61 & CINAHL & ELECTROCARDIOGRAPHY/ & 35246 \\
\hline 62 & CINAHL & (electrocardiograph*).ti,ab & 7423 \\
\hline 63 & CINAHL & (59 OR 60 OR 61 OR 62) & 44870 \\
\hline 64 & CINAHL & $\left(\right.$ electrode $\left.{ }^{\star}\right) . t i, a b$ & 9324 \\
\hline 65 & CINAHL & ELECTRODES/ & 3648 \\
\hline 66 & CINAHL & (64 OR 65) & 11348 \\
\hline 67 & CINAHL & (placement).ti,ab & 34494 \\
\hline 68 & CINAHL & (position*).ti,ab & 79349 \\
\hline 69 & CINAHL & (anatomical).ti,ab & 17470 \\
\hline 70 & CINAHL & (misplacement).ti,ab & 470 \\
\hline 71 & CINAHL & (Angle of louis).ti,ab & 8 \\
\hline 72 & CINAHL & (malposition).ti,ab & 948 \\
\hline 73 & CINAHL & (misposition*).ti,ab & 8 \\
\hline 74 & CINAHL & (mis-position*).ti,ab & 1 \\
\hline 75 & CINAHL & (misplaced OR mis-placed).ti,ab & 640 \\
\hline 76 & CINAHL & $($ error*).ti,ab & 52335 \\
\hline 77 & CINAHL & "HEALTH CARE ERRORS"/ OR exp "DIAGNOSTIC ERRORS"/ & 21009 \\
\hline 78 & CINAHL & (misdiagnos* OR mis-diagnos*).ti,ab & 6531 \\
\hline 79 & CINAHL & (67 OR 68 OR 69 OR 70 OR 71 OR 72 OR 73 OR 74 OR 75 OR 76 OR 77 OR 78) & 198453 \\
\hline 80 & CINAHL & (63 AND 66 AND 79) & 0 \\
\hline
\end{tabular}

An initial screening of titles and abstracts of identified citations was performed by a single reviewer (AH). Potentially eligible articles were then full text screened independently by two reviewers, (AH and SM) in-line with the inclusion and exclusion criteria. Disagreements regarding eligibility were discussed and resolved by a third reviewer (TU). In instances of unclear reporting, authors were contacted to provide further information and clarity.

Assessment of relevant literature and critical appraisal of primary research, pertaining to the clinical diagnosis and effects of anatomical misplacement of ECG electrodes and leads, formulate the thematic discussion drawn by this review.

The primary consideration and rationale for this review is the ongoing potential relevance of electrode misplacement, its frequency in the clinical setting, the subsequent ramifications imposed on patients and reasons leading to such error. In addition, synthesis of the literature correlate the overarching consensus for education, training and a change to practice in order to provide improved safety, and care of patients in line with the Nursing and Midwifery Code of Conduct (NMC, 2015) ${ }^{[22]}$ and guidelines / recommendations of the (SCST, 2017). ${ }^{[19]}$

\section{Study Selection:}

Two independent reviewers (AH and SM) carried out searches of relevant and pertinent studies that met inclusion suitable to this review. Full texts were obtained, with exclusion of obviously irrelevant titles, abstracts, reviews or literature. The independent findings were then cross-examined and assessed against the eligibility criteria. A third reviewer (TU) then made further assessment and agreement, with resolve of any doubt or disagreement prior to the submitted work. English language articles published in indexed journals have been considered along with unpublished works and 'Grey' literature considered.

\section{Database Searches:}

Medline, Embase, CINAHL, were searched, with combinations of subject headings, Boolean terms and Text words agreed from an initial scoping search ensuring a sensitive search. A supplementary search was conducted using NHS evidence and, clinical trials registries, and the international clinical trials registry platform WHO, and the U.K. clinical trials gateway NHIR. This with the view to negate publication bias, whereby published papers tend to show the positive effect of an intervention, rather than the negative effect. ${ }^{[25][26]}$ 
A hierarchy of evidence framework has been used to aid in the evaluation and reduction of bias of included papers, and in acknowledgement of evidencebased healthcare (i.e. Background information/ expert opinion, case-controlled studies, cohort studies, RCTs etc.). In support of this notion, the included papers provide for a broad range of research methods. ${ }^{[27]}$ No restrictions were placed on type of ECG device (manufacturer or model), algorithm or signal processing.

\section{Results}

A total of 3186 articles were identified from the initial search strategy, reducing to 3131 after duplicates were removed. Full text screening was performed utilising NICE Healthcare Database Advance Search (HDAS) ${ }^{[24]}$ was conducted on 78 articles, according to the eligibility criteria, which resulted in 7 included studies, Table 1 (Systematic Review Synthesis and Data Extraction) and Figure 1. PRISMA flow diagram, detailing searches strategy of systematic review.

\section{Data Extraction and Synthesis:}

Five themes were derived from the Systematic Review Synthesis and Data Extraction Table 1. The chief characteristics of included studies and themes derived are as follows:

\section{Theme 1 - Misplacement of Electrodes:}

It is well documented within the literature that misplacement of precordial electrodes is long-standing, continues to remain a common occurrence and finding that, so far, has not been effectively addressed. ${ }^{[13]}$ Of concern, are the subsequent clinical and diagnostic/prognostic ramifications for the patient. ${ }^{[28]}$ The findings of this review support this notion, with V1 and V2 being consistently erroneously misplaced superiorly in the $2^{\text {nd }}$ intercostal space rather than in the $4^{\text {th }}$ intercostal space at the right sternal border and left sternal border respectively. ${ }^{\text {[13][10][29][14]][28] [32] }}$

Potentially harmful treatment and/or therapeutic procedures may ensue. Typically, erroneous electrode placement has the potential to affect and alter ECG

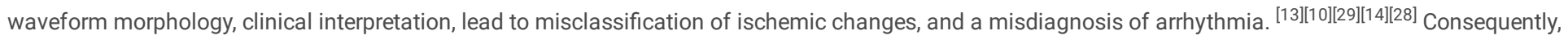
superior misplacement of V1 and V2 spearheads to spurious ECG abnormalities that may include: poor R-wave progression; ventricular hypertrophy; anteroseptal infarct; QRS complex and T-wave alterations; incomplete right bundle branch block (IRBBB); false positive and false negative ischemic changes and Type 2 Brugada syndrome. ${ }^{[30][14][28]}$ A confounding factor and one worthy of consideration is, almost all of the included papers allude to the frequency of misplaced precordial leads V1-V6. [13][10][29][14][28]

Reasons for such erroneous placement are multi-factoral and can include poor training, a lack of appreciation of consequences of misplacement, a failure to understand and utilise anatomical landmarks such as the sternal notch (Manubriosternal joint/Angle of Louis), and the Xiphoid process for correct identification of the $4^{\text {th }}$ intercostal space. ${ }^{[30]}$

Furthermore, levels of undress and the desire to preserve patient modesty, particularly in females, are factors that evidence significant impact. Fewer cardiac investigations are evidenced in women with ACS than in men. Subsequently, such inequities result in less treatment and prove potentially detrimental. ${ }^{[31]}$

\section{Theme 2 - Anatomical Differences:}

Anatomical differences between male and female patients provide additional challenges in electrode placement and accurate ECG acquisition. SCST guidelines and recommendations state, by convention electrodes V4, V5 and V6 ought be placed beneath the breast when breast tissue overlies the correct anatomical positions. ${ }^{[19]}$

A study conducted by Wallen et al, (2013) ${ }^{[31]}$ noted $52 \%$ of women preferred electrodes placed on the breast tissue, deeming it less intrusive, $38 \%$ were indifferent and $10 \%$ preferring under the breast. ${ }^{[31]}$ Review of the wider literature and that of the included studies of this review allude to difficulties, particularly on women with large breast tissue mass and, in obese patients, in accurately identifying bony landmarks as anatomical reference points to aid in the correct placement of electrodes. However, current evidence pertaining to misplacement because of women's breast tissue and obesity is inconclusive and warrants further investigation. ${ }^{[13][30][28]}$

In contrast, a prospective observational study by McCann et al, (2007) ${ }^{[10]}$ evidenced greater lateral chest electrode discordance (V4-V6), irrespective of gender, than those of central chest electrodes (V1-V3), however, identification of anatomical landmarks and accurate location were impeded particularly in older, larger women and the obese. ${ }^{[10][30]}$ This was further supported by the findings of the PULSE multi-site randomised clinical trial, $(2018)$, where $<50 \%$ of both male and female participants were found to have accurate electrode placement. ${ }^{[29]}$ PULSE concluded no significant difference in accuracy for precordial electrode placement between men and women.

\section{Theme 3 - Operator Error \& Causes of Misplacement:}

Inter-operator variability, even among senior clinicians, is a factor widely acknowledged for the occurrence of electrode misplacement. ${ }^{[10]}$ Causes of misplacement extend to both modifiable and non-modifiable patient factors, such as body habitus, body position, lack of anatomical awareness, lack of 
confidence, competence or even over confidence. ${ }^{[10][13]}$ However, confidence is no substitute for competence. ${ }^{[19]}$ Cardiologists in particular fall prey to such over confidence. Assuming the position of V1 and V2 electrodes in the $2^{\text {nd }}$ intercostal space rather than that of the $4^{\text {th, }}$ which may arise from a complacency through regularly listening to auscultations of heart sounds at $2^{\text {nd }}$ intercostal level. ${ }^{[13]}$

It is postulated that particularly amongst paramedics, operator error is compounded by a hesitancy to expose the female breast. The connotation that the female breast is a secondary sexual organ provides ensuing embarrassment. ${ }^{[31]}$

\section{Theme 4 - Clinical Impact on Diagnosis, Treatment \& Management:}

Correlation between misplaced precordial electrodes with changes to ECG morphology dominates the theme of the majority of papers included in this review. Such alterations have the potential to significantly impact clinical interpretation and diagnosis, leading to over, under or even a missed diagnosis. Conversely, superior misplacement of V1 and V2 has proven beneficial in the detection of ST-segment elevation myocardial infarction (STEMI), Non-ST-segment elevation myocardial infarction (NSTEMI) and Brugada syndrome, with no noted significant change to specificity. ${ }^{[28]}$ However, studies have purported a shift in placement by as little as $2 \mathrm{~cm}$; can affect interpretation and diagnosis in as much as $17-24 \%$ of patients. ${ }^{\text {[30] }}$

\section{Theme 5 - Education \& Training:}

Overarching consensus of included papers, place education and training as tantamount, and of paramount importance to accurate ECG acquisition. Thus, optimising sound clinical diagnosis and practice. Implementation and adherence to SCST and the American Heart Association (AHA) guidelines, yields significant improvement. ${ }^{[29][14]}$ Correct identification of anatomical landmarks, notably, the sternal notch (Angle of Louis), Xiphoid process and the $4^{\text {th }}$ intercostal space for the correct placement of electrodes is essential in the undertaking of ECG. This coupled with on-line education and strategies to change current erroneous practice, through raising awareness and knowledge, are fundamental to good clinical practice, quality of care and outcomes for patients, with a reduction in patient morbidity and mortality. ${ }^{[10][29]}$

\section{Discussion}

Types of studies included randomised controlled trials (RCT), systematic reviews, literature reviews and observational studies. Studies were identified irrespective of design, complexity, aims and time of follow-up or hypotheses. Of note, the overriding theme and consensus of the included papers allude to the frequency, commonality and potential for erroneous recordings owing to ECG electrode misplacement. As aforementioned, V1 and V2 are most commonly misplaced anatomically superiorly, with V4 - V6 misplaced inferiorly. The majority of reviewed papers acknowledge the frequency of misplaced precordial electrodes and leads. Subsequently, this results in alteration of ECG waveform morphology, misclassification of ischemic changes and erroneous clinical interpretation. Consensus further points to operator error, with inability to correctly identify intercostal spaces and accurate placement of electrodes, as the overriding underpinning factor for such error. Anatomical differences prove challenging and are factors compounded by obesity and breast tissue mass particularly in women.

\section{Limitations:}

The inclusion criteria for this review included English language articles, international papers, and unpublished works. However, the authors acknowledge that the reviews findings are limited, despite supplementary searching, and that no guarantee can be assigned that all relevant literature has been included. It is further acknowledged that there are inaccessible papers and/ or 'Grey' literature that authors did not have rights over.

\section{Conclusion}

This review has identified that electrode misplacement is well documented, common, ongoing and prevalent throughout healthcare. Misplacement of precordial electrodes is synonymous with erroneous acquisition and significantly alters ECG waveform morphology, leading to misinterpretation and the potential to alter diagnosis. Therefore, correct anatomical placement of the ECG electrodes is in fact essential to diagnosis in the clinical setting. Reasons for misplacement are as aforementioned multifaceted, however the recommendation is that peer-led educational intervention with mandatory training is essential to improve practice and thereby enhance patient safety and outcome.

\section{Key Points And Recommendations}

- Precordial electrodes V1 \&V2 are most misplaced

- Operator error, anatomical differences including obesity and breast tissue mass influence electrode misplacement

- Misplacement of electrodes can lead to a misdiagnosis, a missed diagnosis, under or over diagnosis

- Educational intervention through peer-led training is essential.

\section{Abbreviations}

- ACS: Acute Coronary Syndrome(s) 
- AHA: American Heart Association

- BCS: British Cardiovascular Society

- CAD: Coronary Artery Disease

- CASP: Critical Appraisal Skills Programme

- CWT: Continuous Wavelet Transform

- ECG: Electrocardiogram

- FT: Fourier Transform

- HDAS: Healthcare Databases Advanced Search

- IRBBB: Incomplete Right Bundle Branch Block

- NICE: National Institute for Health and Clinical Excellence

- NMC: Nursing and Midwifery Council

- NSTEMI: Non-ST-segment Elevation Myocardial Infarction

- PICO: Population Intervention Comparison and Outcome Framework

- PRISMA: Preferred Reporting Items for Systematic Reviews and Meta-Analysis Protocols

- RCT: Randomised Controlled Trials

- SCST: Society for Cardiological Science and Technology

- SOB: Shortness of Breath

- STEMI: ST-segment Elevation Myocardial Infarction

\section{Declarations}

\section{- Ethics approval and consent to participate}

'Not Applicable'

\section{- Consent for publication}

'Not Applicable'

\section{- Availability of data and materials}

The datasets generated and/or analysed during the current study are available in the National Institute for Health and Clinical Excellence (NICE), Healthcare Databases Advanced Search (HDAS) (2019) [Online]. Available: http://www.nice.org.uk/ (accessed 25 October 2019) repository.

\section{- Competing interests}

The authors declare that they have no competing interests.

\section{- Funding}

This review has been self-funded.

\section{- Authors' contributions - Author Details:}

$\mathrm{AH}^{1}, \mathrm{SM}^{2}, \mathrm{TU}^{3}, \mathrm{KO}^{4}$

1. Chief Cardiac Physiologist, Royal Cornwall Hospitals NHS Trust, Truro, Cornwall.

2. Interventional Cardiology Registrar, Royal Cornwall Hospitals NHS Trust, Truro, Cornwall.

3. Consultant Cardiologist, Royal Cornwall Hospitals NHS Trust, Truro, Cornwall.

4. Librarian Specialist, Royal Cornwall Hospitals NHS Trust, Truro, Cornwall.

All persons who meet authorship criteria are listed as authors and have participated sufficiently in the work to take responsibility for the content, including participation in the concept, design, analysis, writing, or revision of the manuscript. Outlining everyone's contribution, conception or design performed by Alexandros Hadjiantoni. Data collection was performed by Alexandros Hadjiantoni and Siddhartha Mengi. Article drafting was performed by Alexandros Hadjiantoni and Siddhartha Mengi. Critical revision of the article was performed by Siddhartha Mengi and Tamas Ungvari. Furthermore, each author certifies that this material or similar material has not been and will not be submitted to or published in any other publication.

\section{- Acknowledgements:}


The author extends his gratitude to Dr. Judit Ungvari and librarian specialist, Mrs. Katy Oak and Dr. Tamas Ungvari and Dr. Siddharta Mengi for their insights, and special thanks and guidance from Prof. Muhammad SN.

\section{- Competing Interests:}

There are no conflicts of interest to declare. The authors declare that they have no competing interests.

\section{References}

1. Jones DS, Greene JA. The decline and rise of coronary heart disease: understanding public health catastrophism. Am J Public Health. 2013;103(7):12071218.

2. Fisher EA, Lee J. Coronary Artery Disease. [Internet]. Dallas: American Heart Association; 2015 [cited 2019 Dec 25]. Available from: https:// www.Heart.org

3. Mahmoodzadeh S, Moazenzadeh M, Rashidinejad H, Sheikhvatan M. Diagnostic performance of electrocardiography in the assessment of significant coronary artery disease and its anatomical size in comparison with coronary angiography. J Res Med Sci. 2011;16(6):750-755.

4. Keogh JE, Reed D. Schaum's outline of ECG interpretation. New York: McGraw-Hill; 2011.

5. Klabunde RE. Cardiovascular physiology concepts. 2nd ed. Philadelphia: Wolters Kluwer; 2012.

6. Bayes de Luna A. Clinical electrocardiography: a textbook. 4th ed. Chichester: Wiley-Blackwell; 2012.

7. Kligfield P, Gettes LS, Bailey JJ, Childers R, Deal BJ, Hancock EW, et al. Recommendations for the standardization and interpretation of the electrocardiogram: part I: The electrocardiogram and its technology: a scientific statement from the American Heart Association Electrocardiography and Arrhythmias Committee, Council on Clinical Cardiology; the American College of Cardiology Foundation; and the Heart Rhythm Society: endorsed by the International Society for Computerized Electrocardiology. Circulation. 2007 Mar 13; 115(10):1306-24.

8. Lynch R. ECG lead misplacement: a brief review of limb lead misplacement. Afr J Emerg Med. 2014; 4(3):130-139.

9. Pearce A. Examining the causes and effects of electrode misplacement during electrocardiography: a literature review. Br J Card Nurs. 2019; $14(7)$ : 1-15.

10. McCann K, Holdgate A, Mahammad R, Waddington A. Accuracy of ECG electrode placement by emergency department clinicians. Emerg Med Australas; 2007; 19(5):442-448

11. Kaur I, Rajni R, Marwaha A J. ECG signal analysis and arrhythmia detection using wavelet transform. Inst. Eng. India Ser. B. 2016; 97(4):499-507

12. Goldberger AL, Goldberger ZD, Shvilkin A. Goldberger's clinical electrocardiography: a simplified approach. Philadelphia: Elsevier; 2018. 6 p.

13. Rajaganeshan R, Ludlam CL, Francis DP, Parasramka SV, Sutton R. Accuracy in ECG lead placement among technicians, nurses, general physicians and cardiologists. Int J Clin Pract. 2008; 62(1):65-70.

14. Medani SA, Hensey M, Caples N, Owens P. Accuracy in precordial ECG lead placement: improving through a peer-led educational intervention. J Electrocardiol. 2018; 51(1):50-54.

15. British Cardiovascular Society. Strategic review of cardiac physiology services In England: final report. [Internet]. 2015 [cited 2019].Availablefrom:http://www.bcs.com/documents/SRCPS_Final_Report_12052015_2.pdf

16. National Institute for Health and Clinical Excellence. 4 Developing review questions and planning the evidence review [Internet]. London: National Institute for Health and Clinical Excellence;2014[cited2019].Availablefrom:https://www.nice.org.uk/process/pmg20/chapter/developing-review-questions-andplanning-the-evidence-review

17. Khunti K. Accurate interpretation of the 12-lead ECG electrode placement: A systematic review. Health Educ J. 2014; 73(5):610-623

18. Bond RR, Finlay DD, Nugent CD, Breen C, Guldenring D, Daly MJ. The effects of electrode misplacement on clinicians' interpretation of the standard 12lead electrocardiogram. European Journal of Internal Medicine. 2012; 23(7):610-615.

19. Campbell B, Richley D, Ross C, Eggett CJ. Clinical Guidelines by Consensus: Recording a standard 12-lead electrocardiogram: an approved method by the Society for Cardiological Science and Technology (SCST). [Internet]. 2014 [cited 2019]. Available from:

https://www.bmj.com/sites/default/files/response_attachments/2016/09/CAC_SCST_Recording_a_12lead_ECG_final_version_2014_CS2v2.0.pdf

20. Apoorv G, Kaur, M. ECG analysis using Continuous Wavelet Transform (CWT): IOSR J Eng. 2012 Apr; 2 (4): $632-635$.

21. Critical Appraisal Skills Programme. CASP 12 questions to help you make sense of a cohort study checklist. [Internet]. Oxford:CASP; [2020; cited 2019 December 25]. Available at www.casp-uk.net.

22. Nursing and Midwifery Council. (2015) 'The code': professional standards of practice and behaviour for nurses and midwives. London: Nursing and Midwifery Council.

Page $12 / 13$ 
23. Preferred reporting items for systematic review and meta-analysis protocols (PRISMA-P) [Internet]. Ottawa, Ca:PRISMA; [2015; cited 2019 October 25]. Available at www.prisma-statement.org.

24. National Institute for Health and Clinical Excellence (NICE). Healthcare Databases Advanced Search (HDAS) [Internet]. London:NICE; [2020 July 24; cited 2019 October 25]. Available: http://www.nice.org.uk/

25. Aveyard H. Doing a literature review in health and social care: a practical guide. 3rd ed. Berkshire: Open University Press; 2014.

26. Walker, Sharon (2015). Literature Reviews: Generative and Transformative Textual Conversations [21 paragraphs]. Forum Qualitative Sozialforschung / Forum: Qualitative Social Research, 16(3), Art. 5.

27. Evans D. Hierarchy of evidence: a framework for ranking evidence evaluating healthcare interventions. J Clin Nurs. 2003 Jan; $12(1): 77-84$.

28. Walsh B. Misplacing V1 and V2 can have clinical consequences. The Am J Emerg Med. 2018 May; 36(5): 865-870.

29. Davis LL, Funk M, Fennie KP, May JL, Stephens K, Drew BJ, and PULSE Site Investigators. Does accuracy of V lead electrode placement differ based on gender of patient: results of the practical use of the latest standards of electrocardiography (PULSE) trial. Circulation. 2016 Nov; 134 (suppl 1):A16195.

30. Day K, Oliva I, Krupinski E, Marcus F. Identification of 4th intercostal space using sternal notch to xiphoid length for accurate electrocardiogram lead placement. J Electrocardiol. 2015 August; 48 (6):1058-1061.

31. Wallen R, Tunnage B, Wells S. The 12-Lead ECG in the emergency medical service setting: how electrode placement and paramedic gender are experienced by women. EMJ. 2014 October; 31(10):851-852.

32. Bond, R.R., Finlay, D.D., Nugent, C.D., Moore, G. and Guldenring, D. (2011) A simulation tool for visualizing and studying the effects of electrode misplacement on the 12-lead electrocardiogram. Journal of Electrocardiology [online]. 44 (4), pp.439-444.

\section{Figures}

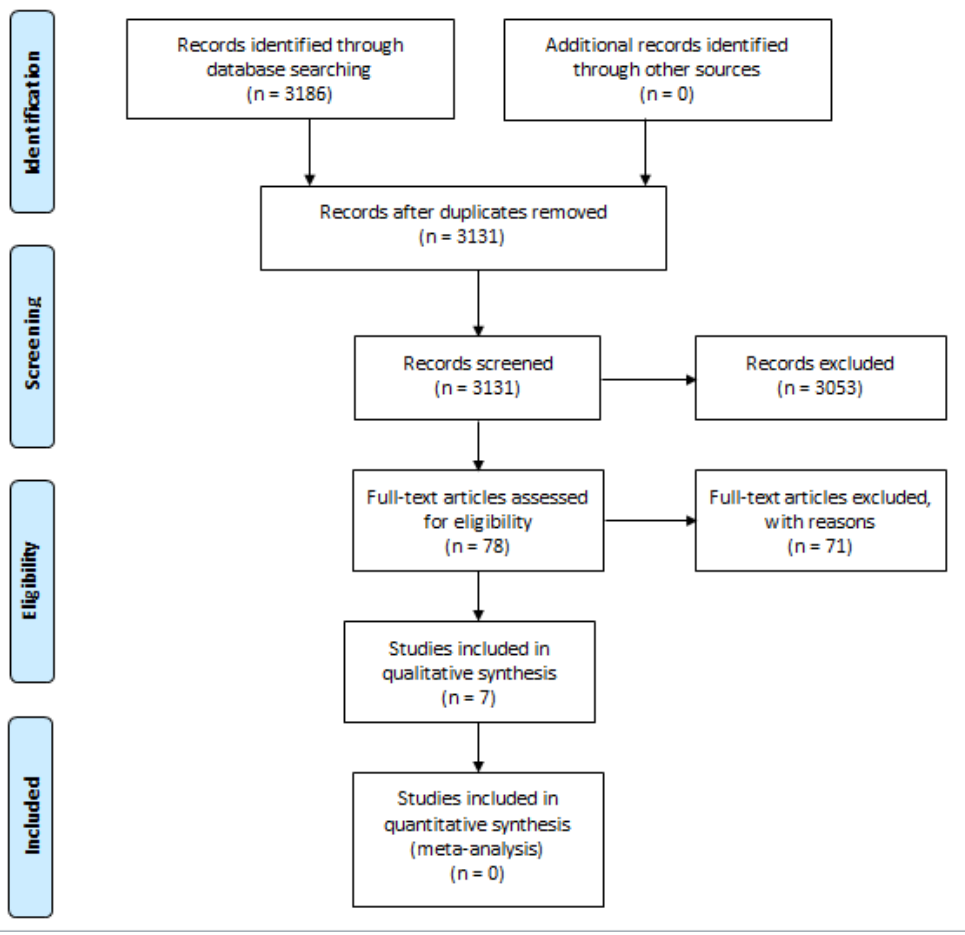

Figure 1

Prisma flow diagram

\section{Supplementary Files}

This is a list of supplementary files associated with this preprint. Click to download.

- PRISMA2009checklistforBMCSpringerNature.doc 